\title{
Rapid Dispatches Highlights
}

\section{3-for-3}

Combinatorial RNA interference (RNAi) is an important approach for researchers who are studying highly mutable viruses such as HIV-1. The ability to express multiple siRNAs from a single genetic construct can be both an effective and efficient technique when studying gene expression, or developing gene therapy strategies directed against viruses. A challenge in engineering these constructs is dealing with the stable intramolecular hairpins associated with short hairpin RNAs which act as stops for DNA polymerase I. Reporting in Rapid Dispatches, Olga Kretova and her colleagues from the Englhardt Institute for Molecular Biology in Moscow, Russia, describe a single step method for the construction of cassettes that simultaneously express three different siRNAs. Their data clearly demonstrated specific and efficient knockdown of the target genes for each of the three siRNAs. The approach, which uses specifically designed oligos that overlap in their loop sequences and PCR to limit hairpin formation, should enable more effective and rapid combinatorial RNAi experiments in the future.

\section{Electric Diatoms}

Our understanding of the basic biological process of some organisms has been hampered by our inability to genetically engineer certain species. One such example of this is the little diatom, an important primary producer in marine ecosystems that has proven difficult to genetically transform. While microprojectile bombardment approaches have allowed for the introduction of exogenous DNA into diatoms, these methods are costly and not common in labs around the world. But as Hong-Ye $\mathrm{Li}$ and colleagues report in Rapid Dispatches this month, the ability to transform diatoms may be possible in any molecular biology lab. It turns out that, under the right conditions and media environment, the commonly used transformation technique of electroporation can be employed to transform diatoms. Li et al. describe the media composition, as well as the electroporator settings, yielding the best transformation efficiencies with diatoms. The authors detail three different plasmids transfected into diatoms, ranging in size

\section{Furry Fluorescence}

Transgenic animals have played a major role in expanding our understanding of biological processes and disease. Transgenic mammals incorporating fluorescent reports often require long periods of time to generate, and genotyping of these animals requires invasive sampling and lengthy protocols. But in a new article published at Rapid Dispatches,

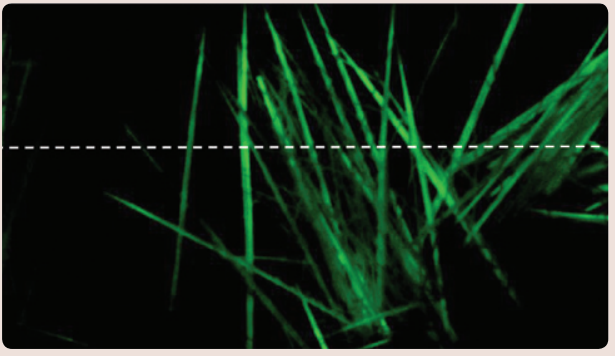

Venus-fluorescence of mouse hair samples, dotted line indicates measured area for intensity histogram. at the Friedrich-Loeffler-Institut in Germany, describe a new, non-invasive approach to genotyping these transgenic animals by imaging hair samples using a fluorescence microscope. Not only were the authors able to determine if an animal expressed the fluorescence marker of interest by imaging the hair samples, but quantitative assessment of fluorescence was also possible and allowed for the rapid differentiation of hemizygous and homozygous status. With the ability to non-invasively sample transgenic mammals and then quickly obtain genotype status, this new technique should find considerable usage in transgenic labs. up to $6.4 \mathrm{~kb}$, and demonstrate the establishment of an inducible selection marker. Taken together, the protocol described by $\mathrm{Li}$ et al. should enable the routine transformation of diatoms, paving the way for genetic engineering within this important marine organism.

\section{Multipurpose Stains}

Amyloids are protein aggregates that have been found to be associated with a number of neurological diseases such as Alzheimers and Creutzfeldt-Jakob disease. Researchers typically identify and study amyloids through the use of a specialized fluorescent dye called thioflavin-T that binds amyloids. Although a common method to study amyloids, thioflavin-T is not uniquely specific for amyloids, and other amyloid binding compounds that have been developed are either non-fluorescent or not available for research purposes. However, an interesting discovery reported in Rapid Dispatches by Steingrimur Stefansson and his colleagues at the Fuzbein Technology Institute in Rockville, Maryland, should greatly expand the amyloid staining toolkit. The authors found that a class of commercially available benzothiazole/benzoxazole containing fluorescent DNA intercalators can bind amyloid peptide and hamster prion. The compounds can act as acceptors in thioflavin-T fluorescence energy transfer experiments as well as a reporter group for binding studies with Congo Red (a non-fluorescent dye that also binds amyloids). Available in a range of fluorescence (500-750 nm), these dyes should find use in the biochemical characterization of amyloids and as tools for in vitro screening of potential therapeutics.

\section{Written by Nathan Blow,Ph.D. [ᄄ由}

Full articles can be found at biotechniques. $\mathrm{com} / \mathrm{rd}$

BioTechniques 52:353 (June 2012)

doi 10.2144/000113875

To purchase reprints of this article, contact:

biotechniques@fosterprinting.com 